Research Article

\title{
Parametric study and Electromagnetic Analysis of Linear Motor for a Stirling Cryocooler
}

\author{
Rajesh V R $\dot{\mathrm{A}}^{*}$ and Biju T Kuzhiveli $\dot{\mathrm{A}}^{\dot{3}}$ \\ ${ }^{\dot{A}}$ Centre for Advanced Studies in Cryogenics (CASC), National Institute of Technology Calicut, Calicut, Kerala, India - 673601.
}

Accepted 10 January 2014, Available online 01 February 2014, Special Issue-2, (February 2014)

\begin{abstract}
The Stirling cycle cooler is a free piston linear motor driven device. The cooling technology using reversed Stirling cycle offers an outstanding opportunity to provide a compact, environment friendly system with comparable energy consumption. Analysis of Stirling cryocoolers has been an important research area for the past many years. This cooler operates as a closed regenerative cycle: closed because there are no valves, a fixed mass of working fluid (usually helium) being repeatedly circulated through the same thermodynamic processes; and it's called as a regenerative cycle as the key component is the thermal regenerator. The linear motor can be considered as the heart of the entire cooler. It drives the compressor piston (or power piston), which compresses the working fluid alternately. In the present work, moving magnet concept has been employed in contrast to the conventional moving coil type motor. This innovative idea avoids the requirements of oscillating electrical connections and reduces the risk of contamination of the working fluid. The permanent magnet material and magnetization, coil gage size and the materials for the inner and outer yoke are important from the point of view of motor performance and cost. The influence of different geometric and electromagnetic parameters on the performance of the motor is analyzed in this paper. For the analysis of each configuration, the relation between the magnet height under the influence of magnetic field and piston amplitude is established mathematically. The periodic variation of active magnet height in the magnetic field has been analyzed electromagnetically and the relations among the parameters are determined.
\end{abstract}

Keywords: Stirling cooler, linear motor, moving magnet.

\section{Introduction}

Even though invented as a hot air engine, Stirling cycle, later, proved its capability in cooling technology as well. Cooling by reversed Stirling cycle provides the most efficient cooling system, as Stirling cycle has the highest efficiency in ideal case. As helium is used as the working medium, owing to its ideal gas properties, high thermal conductivity and high ratio of specific heats, the cooler is an environmentally friendly one also. The Stirling cycle cryocooler offers small size, light weight, high efficiency, low power consumption and high reliability. These advantages make it suitable for military and space applications. For some instruments in spacecrafts, stored cryogens can be completely replaced by closed cycle coolers if a reliable long life system capable of producing a few milli watts of cooling at cryogenic temperatures can be developed. Such a development would enable passively cooled space vehicles to achieve long life and could cool the selective instruments to cryogenic temperature range that can not be achieved by passive cooling alone.

The power piston of the cooler is operated by the motion imparted to it from the linear motor. Linear motors are employed in Stirling coolers, instead of conventional

*Corresponding author: Rajesh V R

DOI: http://dx.doi.org/10.14741/ijcet/spl.2.2014.70 rotary motors. Linear motors generate force in the direction of travel of the piston. They are capable of extremely high speeds, quick acceleration and accurate positioning.

The influence of various geometric as well as electromagnetic parameters on the performance of the motor is to be understood thoroughly in order to make an optimal configuration. Each and every parameter has its own importance in the design and the performance of the entire system, in general. Both radially and axially energized magnet motors are considered for analysis. From the results, the importance of various geometric as well as electromagnetic parameters can be observed.

\section{Linear drive system}

The compressor part of the cooler comprises of the power piston, which compresses the working fluid during one part of the cycle. The piston movement is accomplished by electric motors. The motor may be either rotary type or linear type. In conventional rotary type motors, crank mechanism is employed to obtain reciprocating motion of the piston. In order to reduce the number of moving parts and to eliminate the possibility of wear and tear, linear motors can be employed advantageously. Also there is a 
chance of helium gas contamination by the presence of bearing grease and other motor constituents in rotary types.

The piston and displacer are the only moving components in a Stirling cooler. In a free piston machine, the piston is not rigidly connected to the driving mechanism such as crank. The piston is coupled with the motor that is driven at the supply frequency. The linear motor applies thrust directly to a load, and does not require an intermediate mechanism to convert rotary to linear motion. The displacer is driven by the pressure differential, acting on the rod assembly, created by piston motion and transmitted through the working fluid to maintain the displacer at a constant phase angle to the piston. This piston action, repeatedly compresses and expands the helium working gas, and creates a warm (compression) and a cool (expansion) space. The piston and the displacer are not connected mechanically and are free to move relative to each other. It is nothing but the displacer, which shuttles the helium between the cold and hot end of the machine (via the regenerator).

A number of advantages accrue from the resonant linear drive, viz., negligibly small side loads, easy control of the stroke as it is directly proportional to the drive voltage, simplicity of construction, etc. Since the piston is not rigidly attached to any crank-type mechanism, the stroke of the piston needs to be controlled using a feed back control loop.

The linear motor works on the Lorentz force principle. The force is generated by the interaction between the magnetic field created by the permanent magnet or dc windings and the current carrying conductor. Therefore, the Lorentz force equation can be written mathematically as,

$F=B i l$, where $l$ is the length of the current carrying conductor in the magnetic field (S A Nazar, 2002).
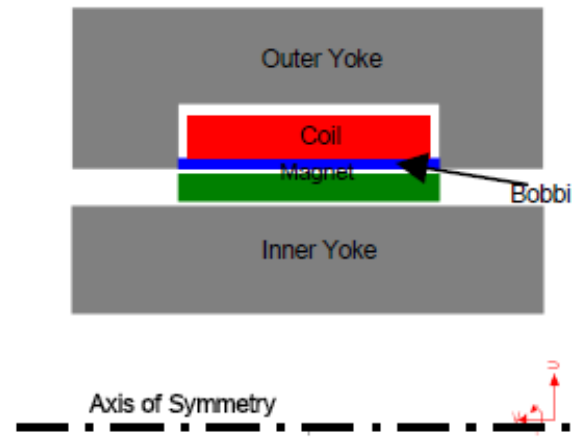

Fig.1 Cross-section of the linear motor configuration

Figure 1 shows the schematic of the cross-section of the linear motor configuration. The cross section is symmetric about the axis of reciprocation. For the present work, a moving magnet type of linear motor is designed and analyzed. The magnet volume required for a moving magnet type is less compared to moving coil for the same output and efficiency. The coil is made stationary in order to eliminate the problems associated with the moving coil type. The moving magnet linear motor offers big advantages over the conventional moving-coil design.
First of all, the coils, known to be a possible source for gas contamination, can be placed outside the hermetically sealed compressor housing containing the working gas. The fact that the coils can be placed outside the hermetically sealed compressor also means that no wire feedthroughs are required. These flying leads have an obvious risk of breaking during their 50 or $60 \mathrm{~Hz}$ operation (i.e., more than 4 million cycles per day!). In this way, risks of feedthrough leakage due to extreme temperature cycles or mechanical shocks are no longer present. Also, as the stationary drive coils are directly mounted to a structure that provides a more effective heat sink; this facilitates the removal of the resistive losses in the coils, and allows for much higher forces. Second, because the coils are stationary, their mass does not affect maximum velocity, permitting the use of a larger (more efficient) coil. Third, with stationary coils, there are no flying leads; this improves motor longevity. Finally, as with all linear motors, the moving magnet does not contact any of the motor's stationary elements, so there is none of the frictional wear associated with mechanical power transmission (R Z Unger et al, 1996).

Like rotary motor, linear motor also has rotor and stator counterparts. In a moving magnet design, magnet replaces the rotor part. The permanent magnets (PM) are used instead of $\mathrm{dc}$ windings to provide a constant magnetic flux field. The radially magnetized ring is placed between the internal and external yokes made of the magnetically soft iron and shaped to accommodate the radial coil carrying $\mathrm{AC}$ current and to provide for the uniform distribution of the magnetic flux within the working air gap without over-saturating the yoke. Placing steel shelling outside the coil allows the magnetic flux, which emanates from the surface of the PM, to be evenly distributed within the effective coil area. Consequently, every turn of coil is fully utilize to generate the desired output force. In a motor of this kind, the interaction of the permanent magnetic field produced by the radially magnetized ring with an alternating electromagnetic field yields the axial force which is applied directly to the movable ring. The ring is rigidly attached to the compression piston which is arranged to slide freely inside the tightly matched cylinder sleeve. This causes the desired movement of the compressor piston. The various components are depicted in figure 1 itself.

\section{Parametric study}

In the theoretical analysis of the motor, the geometrical parameters which are important in the performance analysis of the motor are: magnet height $\left(\mathrm{h}_{\mathrm{m}}\right)$, coil height $\left(h_{c}\right)$, gap height $\left(h_{g}\right)$, the active magnet height $\left(h_{v}\right)$ and the air gap between the outer and inner iron (b). These parameters are marked in a configuration with outer yoke teeth, shown in figure 2 below. In a configuration, where the ratio of gap length to gap width is large, a uniform magnetic field is established across the gap. Within the gap, the magnet can reciprocate as a result of the applied alternating current to the coil. The centre position of the piston-magnet assembly is held and controlled by flexure springs. The configurations can be varied by varying the 
height of magnets, coil, yoke teeth, magnetization of the magnet etc.

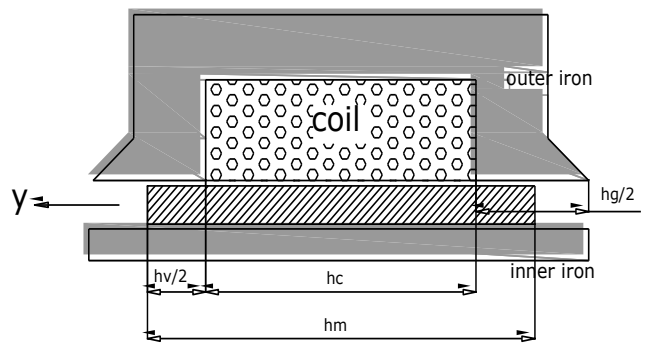

Fig. 2 Geometric parameters of the linear motor

Every magnetic structure consists of a magnet and an iron return circuit for the magnetic flux. The coil is wound circumferentially in the stator. When the coil is wound circumferentially, the magnet do not leave air gap and losses will be minimal. The magnet is directly connected to the moving member, piston, via a carrier.

In any magnetic circuit involving PMs, the magnetic flux it can provide in the air gap matters. The physical dimension of PM and air gap give the exact value of flux density. The working air gap of the magnetic circuit is axially quite long, roughly equivalent to the axial length of the Nd-Fe-B magnet ring. The air gap magnetic field is usually in the range of $0.4-0.7$ times the remanence $\left(B_{r}\right)$ value of the magnet material employed. A PM is characterized by its demagnetization curve or second quadrant property. In the case of Nd-Fe-B PM, it is almost linear and can be expressed as a linear function (Morcos, 2000).

The active magnet height, $h_{v}$, is the height of the magnet under the direct influence of magnetic field at any point of time. The gap height, $h_{g}$, and the coil height, $h_{c}$, are constants. But at the same time the height of the magnet in the field, $h_{v}$, can vary from time to time. The periodic variation of $\left(h_{v} / h_{g}\right)$ with respect to time poses the difficulty in the optimization process. Two different cases could be studied and analyzed in detail for each configuration selected, according to whether the magnet is shorter or longer than the air-gap height or coil height.

For the configuration shown in figure 2 , the mathematical expression for the active magnet height to air gap height ratio would be obtained as

Case 1: when $h_{m}>h_{c}$

$$
\begin{aligned}
& \frac{h_{v}}{h_{g}}=\frac{|y|}{h_{g}}-\frac{h_{c}-h_{m}}{2 h_{g}} \quad \text { for which }|y|>\frac{h_{c}-h_{m}}{2}, \\
& \frac{h_{v}}{h_{g}}=1 \quad \text { for which }|y| \leq \frac{h_{c}-h_{m}}{2}
\end{aligned}
$$

Case 2: when $\mathrm{h}_{\mathrm{m}} \leq \mathrm{h}_{\mathrm{c}}$

$$
\begin{array}{ll}
\frac{h_{v}}{h_{g}}=0 & \text { forwhich }|y| \leq \frac{h_{c}-h_{m}}{2} \\
\frac{h_{v}}{h_{g}}=\frac{|y|}{h_{g}}-\frac{h_{c}-h_{m}}{2 h_{g}} & \text { for which }|y|>\frac{h_{c}-h_{m}}{2}
\end{array}
$$

\section{Electromagnetic analysis}

The principle of electromagnetism is the basis for design of linear motor. The design starts from the electromechanical energy conversion, i.e., the conservation of energy principle. Electromechanical energy conversion occurs when air gap fields are disturbed in such a way that the energy stored in the fields changes with mechanical motion. The force generated by the interaction of magnetic fields of coil and permanent magnet can be obtained by equivalent magnetic circuit analysis.

The electromagnetic analysis is performed using Ansoft Maxwell 11.0. This software solves the problem using finite element method. The magnetic field distribution can be plotted from the results. The flux density in the air gap, which is produced mainly by the PM influences the back EMF generated by the motion of the magnet. The air gap flux density distribution and the leakage fluxes are also obtained from the analysis. The analysis confirms the motion of the PM in the axial direction. Even though the particular shape (Figure 2) of the outer iron reduces the eddy current loss, there exists weak leakage flux at both the ends of the yoke.
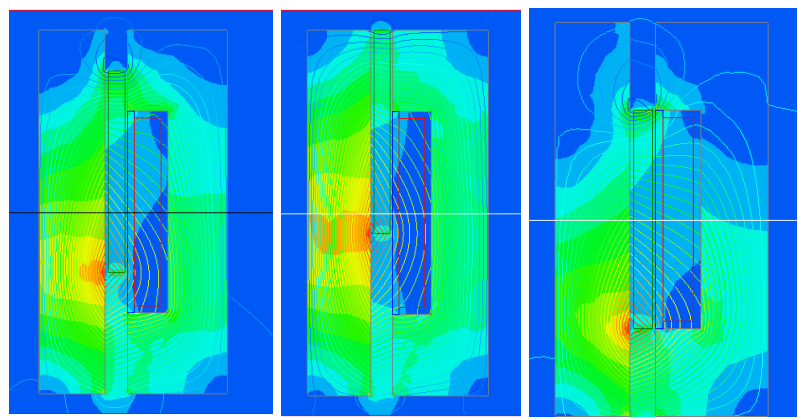

Fig. 3 Magnetic flux distribution

The magnetic flux distribution for a configuration under analysis is shown in the figure 3 above. Three different positions are shown based on the position of the magnet from the centre (balanced) position to the extreme ends. It is found that the magnetic field in the air gap is the superposed fields of the PM and the exciting coil. The maximum flux density in the air gap is almost equal to the one obtained by calculation. As the flux lines are not parallel at the ends of the PM, there exist both radial and axial components of force. Axial component is the useful part and the radial component counteracts circumferentially. Even though the particular shape of the outer iron reduces the eddy current loss, there exists weak leakage flux at both the ends of the yoke. Usually the magnet field is distributed unevenly, making the magnet leakage of the ending large; especially when the mover position changes, the leakage becomes more complex. It can be seen that at different positions, the winding flux obviously differ and the difference produces axial thrust (Z-direction). When two magnet rings are used at both ends, the inner core is subjected to saturation, unless a very large radial thickness for the structure is provided.

\section{Results and Discussion}

The air gap flux density influences the back emf produced 
and thereby the force generated by the motor. Variation of force with respect to piston stroke and for varying thickness of PM are analyzed and presented in the form of characteristic curves as described below.

\subsection{Force vs stroke}

The following graph gives the relation between the axial force developed in the moving magnet motor and the stroke length. The stroke length is considered on either sides of the motor from the mean position of the magnet. For different positions of the magnet the force generated is different. This is because of the difference in the interaction of magnetic field of magnet and the current carrying coil. The figure 4 shows the graph for the selected configuration as discussed in the previous pages.

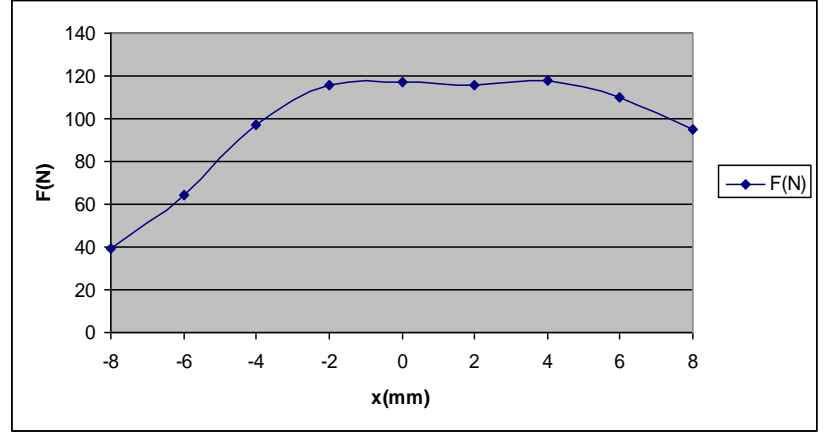

Fig. 4 Force vs Stroke

The maximum force is obtained when the magnet is near the balanced position (centre). This clearly states that when the magnet is under the direct influence of the current carrying coil or nearby it, the maximum force is generated. When the magnet moves away from the center position, the interaction of the magnetic fields (of coil and PM) vary and hence the force too. It is also observed that based on the direction of current supply in the coil, the force direction also changes.

\subsection{Force vs thickness of magnet}

The analysis has been carried out for varying magnet thickness and the results are summarized as in figure 5 . The minimum force obtainable for the given magnet thickness from the motor are plotted herein. The following observations are made from this.

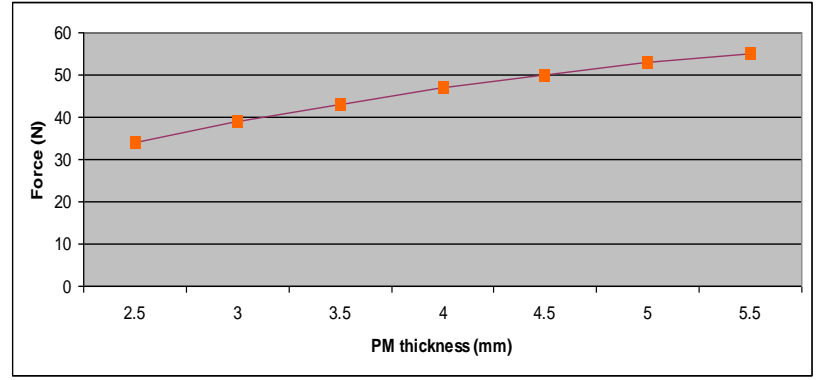

The magnet thickness chiefly affects the Z-direction force. Generally the force can be gradually increased with the increase of the magnet thickness. But the increase in magnet thickness increases the overall weight of the system. Also the increase of the magnet mass leads to the gradual decrease of the dynamic response and further cripples the performance of the cryocooler.

\section{Conclusions}

The linear motor offer flexibility in operation in a number of ways. Firstly, it places the coils, which are main sources of gas contamination, outside the working gas. Also, as no flexible feed throughs for the wires are required, leakage through it is eliminated. The Nd-Fe-B magnets selected are very light in weight with highest energy product available, and hence this cooler is most suitable for weight sensitive applications. As the piston amplitude varies proportional to the input voltage, the control of the system is also easy. The parametric study and electromagnetic analysis results obtained show the feasibility of linear motor to impart acceleration to the piston of the Stirling cooler. The finite element analysis is carried out to obtain the magnetic flux distribution and flux density. The results such as magnetic field distribution, variation of axial force with thickness of PM and stroke length are obtained. It is thereby concluded that the motor with teeth (slots) has smaller air gap, which helps in achieving larger air gap flux density.

\section{References}

R.Z.Unger, N.van der Walt (1996) Linear compressors for non$\mathrm{CFC}$ refrigeration, International Appliance Technical Conference.

Tony Morcos (2000), The straight attraction, Motion Control, The Magnequench technology center.

Poornima, Hsu (2001), Moving magnet loudspeaker system with electronic compensation, IEE Proceedings - Circuits Devices Systems, Vol. 148, No.4, pp 211-216.

$\mathrm{M}$ Linde et al (2001), Cryogenics in space - a review of the missions and technologies, European Space Agency bulletin 107.

R. Mikalsen, A.P. Roskilly (2005), A review of free-piston engine history and applications, Applied Thermal Engineering, Volume 27

S.A.Nasar (2002), Electric machines and power systems, Tata McGraw-Hill edition.

Magcraft (2007), Advanced magnet selection and design handbook.

Fig. 5 Force vs Magnet thickness 